\title{
Genetics \\ and Sports
}

\section{2nd, revised and extended edition}

\section{Editors: Michael Posthumus Malcolm Collins}

This second edition of Genetics and Sports expands on topics previously discussed in an attempt to create an integrated and holistic understanding of the field of sports genomics. It is an update on technologies and on the role of genetics in training, performance, injury, and other exercise-related phenotypes. Ethical concerns and the importance of counselling before and after genetic testing are also addressed.

It is increasingly important to understand the field of genetics and sports because of the potential to use and misuse information. All exercise scientists, sport and exercise clinicians, athletes, and coaches need to be adequately informed to ensure that genetic information is accurately and properly used. Genetics and Sports is, therefore, highly recommended to all of these groups.

\section{CONTENTS}

Preface: Posthumus, M.; Collins, M.

Core Concepts in Human Genetics: Understanding the Complex Phenotype of Sport Performance and Susceptibility to Sport Injury: Gibson, W.T.

Nature versus Nurture in Determining Athletic Ability: Yan, X.; Papadimitriou, I.; Lidor, R.; Eynon, $\mathrm{N}$.

Recent Research in the Genetics of Exercise Training Adaptation: Venezia, A.C.; Roth, S.M.

Genes and Athletic Performance: An Update: Ahmetov, I.I.; Egorova, E.S.;

Gabdrakhmanova, L.J.; Fedotovskaya, O.N.

The Future of Genomic Research in Athletic Performance and Adaptation to Training: Wang, G.; Tanaka, M.; Eynon, N.; North, K.N.; Williams, A.G.; Collins, M.; Moran, C.N.; Britton, S.L.; Fuku, N.; Ashley, E.A.; Klissouras, V.; Lucia, A.; Ahmetov, I.I.; de Geus, E.; Alsayrafi, M.; Pitsiladis, Y.P.

Genes and Musculoskeletal Soft-Tissue Injuries: Rahim, M.; Collins, M.; September, A. Genetics of Musculoskeletal Exercise-Related Phenotypes: Collins, M.; O'Connell, K.; Posthumus, M.

Genetic Testing for Sports Performance, Responses to Training and Injury Risk: Practical and Ethical Considerations: Williams, A.G.; Wackerhage, H.; Day, S.H.

Author Index

Subject Index

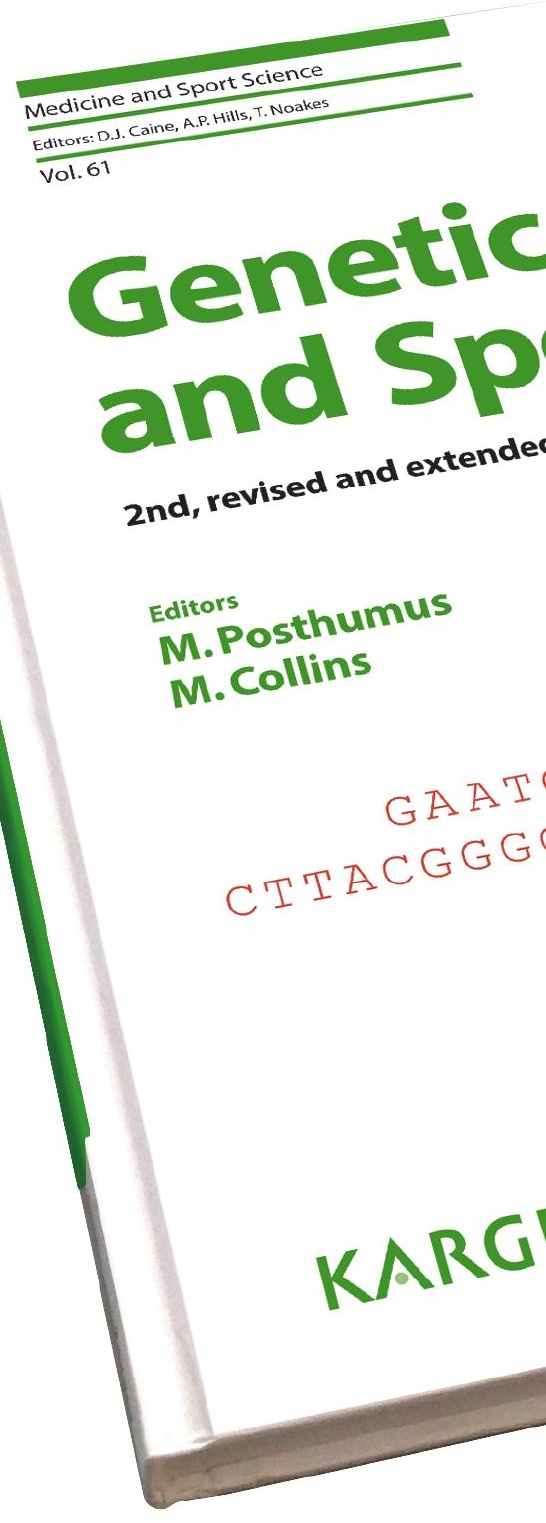




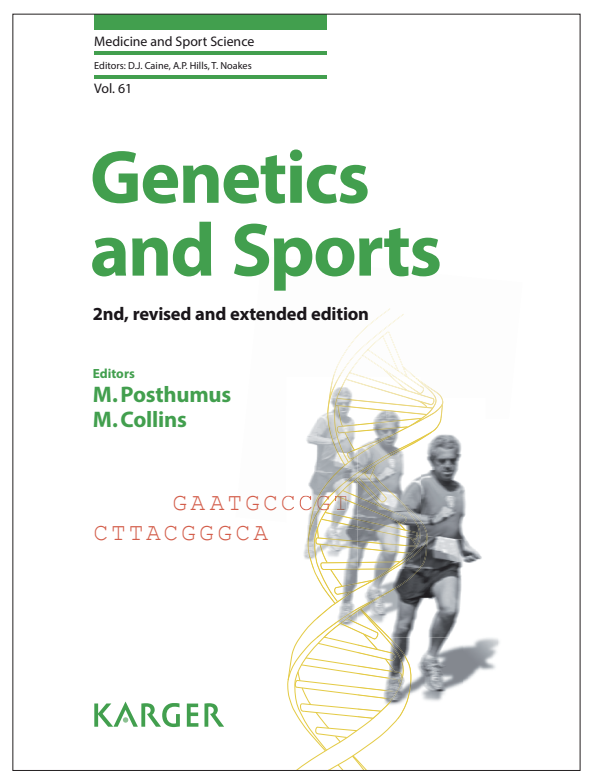

Medicine and Sport Science, Vol. 61

\section{Genetics and Sports}

2nd, revised and extended edition

Editors: Posthumus, M.; Collins, M. (Cape Town)

VIII + 124 p., 2 fig., 6 tab., 2016

CHF 132.00 / EUR 123.00 / USD 155.00

(hard cover or online*)

CHF 158.00 / EUR 148.00 / USD 186.00 (online**)

ISBN 978-3-318-03010-5

e-ISBN 978-3-318-03011-2

\section{Medicine and Sport Science}

Series Editors: Caine, D.J. (Grand Forks, N.Dak.); Hills, A.P. (Brisbane, Qld.); Noakes, T. (Cape Town)

ISSN 0254-5020 / e-ISSN 1662-2812

Listed in MEDLINE/Pub Med

\section{FIELDS OF INTEREST}

Sports Medicine, Genetics, Musculoskeletal Systems; Orthopedics, Physiology, Molecular Biology,

Genomics, Human Genetics

\section{Further publications in the field}

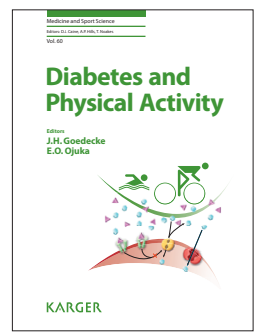

Vol. 60

Diabetes and Physical Activity

Editors: Goedecke, J.H.; Ojuka, E.O. (Cape Town)

VIII + 158 p., 15 fig., 8 tab., hard cover, 2014

CHF 189.00 / EUR 177.00 / USD 222.00 (hard cover or online*)

CHF 227.00 / EUR 212.00 / USD 266.00 (online (**) $^{* *}$

ISBN 978-3-318-02576-7

e-ISBN 978-3-318-02577-4

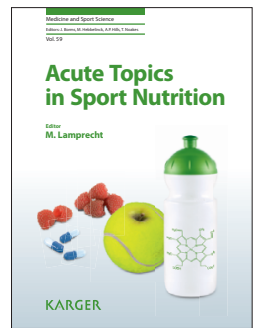

Vol. 59

Acute Topics in Sports Nutrition

Editor: Lamprecht, M. (Graz)

VIII + 156 p., 17 fig., 6 tab., 2012

CHF 189.00 / EUR 177.00 / USD 222.00 (hard cover or online*)

CHF 227.00 / EUR 212.00 / USD 266.00 (online ${ }^{* *}$ )

ISBN 978-3-8055-9992-4

e-ISBN 978-3-8055-9993-1

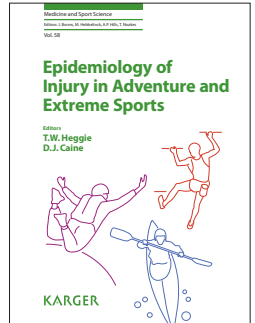

Vol. 58

Epidemiology of Injury in Adventure and

Extreme Sports

Editors: Heggie, T.W. (Bowling Green, Ohio);

Caine, D.J. (Grand Forks, N.Dak.)

$\mathrm{VI}+178$ p., 18 fig., 46 tab., 2012

CHF 195.00 / EUR 182.00 / USD 229.00 (hard cover or online*)

CHF 234.00 / EUR 218.00 / USD 275.00 (online ${ }^{* *}$ )

ISBN 978-3-318-02164-6

e-ISBN 978-3-318-02165-3

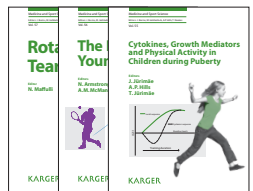

Vol. 1-57 available, please ask for details

\section{www.karger.com/mspsc}

Orders can be placed at agencies, bookstores, directly with the publisher, or with any Karger distributor.

S. Karger AG, P.O. Box, 4009 Basel (Switzerland) e: orders@karger.com, f: +41 613061234

USA: S. Karger Publishers, Inc., 26 West Avon Road, P.O. Box 529, Unionville, CT 06085, Toll free: +1-800-828-5479

Germany: S. Karger GmbH, 79095 Freiburg

France: Enter \& Read, Albertine Luginbuhl, 75014 Paris

Japan: Karger Japan, Inc., Tokyo 105-0012

South East Asia, China and Taiwan: Karger Regional Office (Malaysia),

46050 Petaling Jaya, Selangor Darul Ehsan

Argentina, Brazil, Chile, Mexico, Uruguay: dot.lib, 01418-000 São Paulo

India, Bangladesh, Sri Lanka: Karger India, New Delhi 110016

Thailand: S. Karger, Nakhon Pathom 73170

Russian Federation: World of Periodicals, Moscow 125190

South Eastern Europe (w/o Greece) - for books only: Dr. László Horváth, 1047 Budapest

For details and further representatives and agencies see:

www.karger.com/worldwide
Library Recommendation

Dear Librarian

I have reviewed Genetics and Sports and would like to recommend it for your library

Recommended by:

**online prices for institutional purchas

Prices subject to change, VAT not included.

EUR price for eurozone countries, USD price for USA and Latin America only

amazon Google play Vitalsource

ebooks are available on

Department:

Date:

Signature: 